\title{
Abordaje sistémico en familias latinas sobrevivientes de violencia doméstica en Estados Unidos
}

\section{Systemic Approach in Latino Families Surviving Domestic Violence in the United States of America}

\author{
Claudia B. García-Leeds ${ }^{1 *} \&$ Liora Schneider ${ }^{2}$
}

Chestnut Hill College, Filadelfia, Estados Unidos.

${ }^{1}$ Profesora Asistente en Chestnut Hill College. Doctorado en Psicologia Clinica. ${ }^{2}$ Instructora en Chestnut Hill College. Maestria en Ciencias

\author{
Recibido 30-06-17 \\ Aprobado 14-08-17 \\ En Línea 17-08-17
}

*Correspondencia

Email: garcialeedsc@chc.edu

\section{Citar como:}

Garcia-Leeds, C., \& Schneider L. (2017). Abordaje sistémico en familias latinas sobrevivientes de violencia doméstica en Estados Unidos. Propósitos y Representaciones, 5(2), 277 - 319. doi: http:// dx.doi.org/10.20511/pyr2017.v5n2.164

\footnotetext{
(c) Universidad San Ignacio de Loyola, Vicerrectorado de Investigación, 2017.

(cc)BY-NC-ND Este artículo se distribuye bajo licencia CC BY-NC-ND 4.0 Internacional (http://creativecommons.org/licenses/by-nc-nd/4.0/).
} 


\section{Resumen}

El objetivo de este estudio es crear un modelo de tratamiento sustentado por el conocimiento de profesionales y que les permita el desarrollo de habilidades de los profesionales para su trabajo con las familias en crisis. Las participantes, profesionales de la salud del Latina Domestic Violence Program (LDVP) en Filadelfia, fueron entrevistadas individualmente y las respuestas fueron analizadas de acuerdo con la metodología de la Teoría Fundamentada. Las respuestas de las participantes sirvieron para enfatizar aspectos importantes relacionados con la situación de supervivientes de violencia doméstica. Las participantes identificaron dos elementos centrales necesarios para un programa ideal. 1: El programa debe tener la capacidad de abordar las necesidades biopsicosociales de la familia (necesidades económicas, laborales, educacionales y de salud mental). Las necesidades de las usuarias indocumentadas que no hablan inglés deben ser consideradas en la elaboración de un programa para familias latinas. 2: Es importante que el equipo de trabajo esté entrenado en varias teorías y/o modelos adecuados de intervención que ayuden a una comprensión de la cultura latina de las usuarias. De esta forma se evita utilizar un modelo basado en la cultura estadounidense que no responde a las necesidades específicas de la comunidad.

Palabras clave: Violencia domėstica, programas de intervención, latino, salud mental.

\section{Summary}

This study is aimed at creating a treatment model grounded by professionals' knowledge and that allows the development of skills to work with families in crisis. The participants, health professionals of the Latina Domestic Violence Program (LDVP) in Philadelphia, were interviewed individually, and the answers were analyzed in accordance with grounded theory methodology. The answers of the participants were used to emphasize the important 
aspects related to the situation of survivors of domestic violence. The participants identified two main elements necessary for an ideal program. 1. The program must have the capacity to address all the biopsychosocial needs of the family (financial, occupational, educational and mental health needs). Undocumented and non-English speaking users' needs must be considered when developing a program for Latino families. 2. It is important that the work team should be trained in several appropriate theories and/or of intervention that help to understand the Latino culture of the users. In this way, the use of a model based on the American culture that does not meet the specific needs of the community is avoided.

Keywords: Domestic violence, intervention programs, Latino, mental health. 


\section{Introducción}

El objetivo de este estudio es desarrollar un modelo efectivo para entender y tratar a la violencia domestica en las comunidades Latinas de la ciudad de Filadelfia. A través del trabajo en Latina Domestica Violence Program (LDVP), una organización que provee servicios sociales a la comunidad latina de Filadelfia, surgió la necesidad de investigar teorías y modelos diseñados para atender a familias latinas que sufrían de violencia doméstica; los modelos existentes no respondían a las necesidades de esta población en particular. Anteriormente, la visión acerca de la violencia domestica, se enfocaba únicamente en el agresor o en la victima. De acuerdo a Varea y Castellanos (2006) un abordaje sistémico será más efectivo en el tratamiento de la violencia domestica.

Después de una exploración extensiva sobre los modelos teóricos utilizados para entender la violencia domestica, Varea y Castellanos (2006) concluyeron que es necesario utilizar un enfoque global que explique el origen y el mantenimiento de la violencia domestica. La violencia domestica será mejor entendida a través de la combinación de varias teorías como el modelo ecológico y la perspectiva de genero (Alencar-Rodrigues \& Cantera, 2012).

Es por esto, que se necesitaba un abordaje más amplio como la teoría fundamentada para empezar el estudio. De acuerdo a Villarejo-Aguilar (2013), la teoría fundamentada es un diseño de investigación cualitativa que se emplea en la creación de teorías sobre la salud. A través de una pregunta global, la teoría fundamentada genera conceptos y crea hipótesis. "La teoría fundamentada no es la voz de los participantes, sino una abstracción generada de sus haceres y significación que se toma como datos para la generación conceptual" (Villarejo-Aguilar, 2013, p. 1).

La metodología de investigación cualitativa fue seleccionada debido a que ofrece la mejor perspectiva de cómo utilizar el "conocimiento local" de las consejeras. Graham-Bermann y Edleson (2001) sugieren que un 
abordaje cualitativo es la forma más efectiva de estudiar problemas como la violencia doméstica, en donde una comprensión multisistémica es necesaria. Para los fines de este estudio, el rol de las consejeras fue crucial ya que dado su contacto con las usuarias, podían aportar información de utilidad, estableciendo el primer contacto con las usuarias y comprendiendo los efectos de la violencia domestica.-

A lo largo del presente estudio se describe el termino de violencia domestica. A través del análisis bibliográfico realizado por Rodríguez Franco, López-Cepero y Rodríguez Díaz (2009), el termino de violencia domestica es el mas común para describir la violencia en la pareja, a pesar de los múltiples conceptos utilizados.

De acuerdo a Corsi (2010), la violencia domestica se presenta en contextos privados en la relación de pareja. Se manifiesta a través del control y dominio sobre la mujer para mantener el poder del hombre en la relación. Se expresa a través de maltrato físico, abuso sexual, económico y ambiental, así como a través del maltrato verbal y psicológico. Las consecuencias son: daño en la salud física, psicológica y social de la mujer.

\section{Método}

Este estudio utilizo tres marcos de referencia para explorar, organizar y explicar el problema de violencia doméstica en la comunidad latina en los Estados Unidos. 1. La teoría ecológica de Bronfenbrenner (1979) sirvió para explicar que la conducta violenta dentro de la familia es el resultado de la interacción entre diversas variables. 2. La metodología de investigación cooperativa (Reason \& Heron, 1995) sirvió para reportar como se obtuvieron los datos. 3. El método de comparación constante (Charmaz, 1995) fue utilizado para analizar los datos y desarrollar un modelo para proveer servicios a las familias latinas en la comunidad. De acuerdo a la teoría ecológica de Bronfenbrenner (1979), las preguntas fueron diseñadas para 
obtener una visión holística de los retos encontrados por las mujeres en la búsqueda de ayuda para lidiar con la violencia domestica.

La metodología de investigación cooperativa resultó el marco de referencia ideal para estudiar el problema de la violencia doméstica en la comunidad latina debido a que reflejó un proceso continuo que se estableció entre el investigador principal y el resto del equipo dentro de LDVP. Este tipo de investigación promueve un ciclo sistemático de "acción y reflexión" que es parte de la dinámica natural dentro del programa. Bausela, (2003) plantea que la investigación cooperativa es una metodología que se enfoca en el cambio planificado y se fundamenta en la investigación-acción llevando a cabo los siguientes pasos: reflexionar antes, durante y después de la acción para planificar y aplicar acciones de mejora y recoger evidencias.

En este abordaje orientado al trabajo en equipo, de acuerdo a la investigación cooperativa, los miembros fueron evaluados de acuerdo a sus roles. Esto originó una reflexión continua (vía supervisión y reuniones en equipo) de los servicios que la agencia proveía a la comunidad.

\section{Participantes}

Participantes: Cinco consejeras y dos supervisoras de LDVP. La permanencia de las consejeras en el programa variaba de seis meses a doce años. La media fue de 4.8 y la desviación estándar de 3.5. Cinco de las siete las participantes eran capaces de comunicarse efectivamente con las usuarias; las dos restantes podían mantener una conversación básica. Cuatro de las participantes eran originarias de Puerto Rico y las tres restantes nacieron en Estados Unidos; una de ellas, hija de padres portorriqueños. También existía un rango de grados académicos dentro del equipo que variaba entre desde preparatoria hasta maestría. Algunas de las participantes eran sobrevivientes de violencia doméstica e inicialmente la relación con el programa fue en calidad de usuarias. Para su inclusión en la muestra se requería ser empleada 
en el programa de LDVP y proveer servicios directos a las usuarias. No hubo criterios de exclusión.

\section{Tabla 1.}

Datos de las participantes.

\begin{tabular}{lcccc}
\hline Nivel Educativo & Preparatoria & Nivel técnico & Bachillerato & Maestría \\
& 1 & 2 & 2 \\
\hline Fluidez En Espanol & Fluida & Conversación básica & & \\
& 5 & 2 & & \\
Lugar De Origen & Puerto Rico & Estados Unidos & & \\
& 4 & 3 & \\
\hline
\end{tabular}

\section{Procedimiento.}

El procedimiento, basado en el método cualitativo, se llevo a cabo a través de entrevistas estructuradas con una duración de una hora o de una hora y media. De acuerdo a Torrecilla, (n.d.), el investigador lleva a cabo una planificación previa de todas las preguntas que formulara. Prepara con anterioridad una batería de preguntas que se coordinan con un guion realizado de forma secuenciada y dirigida.

Todas las entrevistas fueron conducidas por la investigadora principal y fueron grabadas en audio para facilitar la transcripción y traducción de los datos. Las participantes eligieron el idioma en el que se sintieron más cómodas para responder un cuestionario cuya meta fue obtener la opinión de las participantes en relación a los servicios ofrecidos por el programa y saber si éste respondía a las necesidades de las familias.

\section{Análisis de datos.}

El objetivo principal de este estudio fue desarrollar un enfoque informado culturalmente para entender y tratar la violencia doméstica que afecta a la comunidad latina en Filadelfia. Aunque el objetivo principal de este proyecto no era desarrollar una teoría, el proceso de diseño cualitativo es ideal para 
crear modelos que provean servicios más efectivos a la comunidad. Todos los datos recopilados durante las entrevistas fueron transcritos y analizados de acuerdo a la Teoría Fundamentada.

El proceso recomendado por Charmaz (1995) implica examinar línea por línea lo que se ha observado y nombrar cada situación o conducta especifica. Ella explica que este procedimiento tiene varios propósitos: a) mantiene al investigador en contacto cercano con los datos y le impide sacar conclusiones que no estén basadas en hechos. Como debe mantener la atención en los datos, es menos probable que el investigador proyecte su cosmovisión en las respuestas de los participantes. b) La codificación de línea por línea aumenta la probabilidad de que el investigador experimente lo ordinario y mundano en una nueva forma ya que crea distancia entre los encuestados y las ideas preconcebidas.

\section{Resultados}

Los resultados se presentan en cinco secciones: 1) descripción del programa 2) necesidades biopsicosociales de las usuarias 3) limitaciones relacionadas con el sistema y con el programa 4) descripción de la comunidad y sus valores. 5) características esenciales de un programa ideal.

\section{Descripción del programa.}

En el momento del estudio, el equipo en LDVP estaba conformado por 5 consejeras y 2 supervisoras. Las consejeras se ocupaban de la mayoría de los servicios relacionados con las usuarias. Las supervisoras eran responsables de la intervención en crisis, hacer guardia de ser necesario y de asegurarse de que los servicios fueran provistos de acuerdo a las entidades regulatorias. 


\section{Consejería.}

Las consejeras proveían varios servicios a las familias, trabajaban con otros sistemas en favor de las usuarias en el contexto de la violencia doméstica, apoyaban a las usuarias en cuestiones relacionadas con otras agencias y daban información a usuarias indocumentadas acerca de las leyes y los servicios disponibles. Desafortunadamente, la falta de fondos dificultó que se ofrecieran servicios de consejería más específicos a la comunidad.

\section{Manejo de casos de las consejeras y supervisoras.}

Las consejeras hablaron acerca de los diferentes marcos de referencia utilizados para asegurar que el manejo de casos/consejería fuera terapéutico y empoderado y que facilitara el establecimiento de una relación de colaboración y confianza entre la usuaria y las proveedoras de servicio.

La situación de violencia doméstica en la usuaria junto con numerosos estresores psicosociales causaba un estado perpetuo de crisis. Las usuarias acudían inicialmente porque necesitaban saber qué hacer después de una situación violenta con su pareja. Sin embargo, la crisis no disminuía cuando los problemas de la relación eran resueltos, ya sea proveyendo a la usuaria con un plan de seguridad o facilitándole la reubicación. De hecho, mientras que las consejeras evaluaban las opciones de las usuarias, aparecían otras necesidades significativas como falta de documentos legales o acceso a servicios médicos por lo que las situaciones de crisis eran constantes.

Además de los servicios de consejería, manejo de casos, intervención en crisis y derivación a otros servicios de referencia, se proveían a la comunidad servicios adicionales como la línea de ayuda en crisis para dar asistencia y talleres educacionales relacionados con violencia doméstica. Dado el número de necesidades identificadas por el equipo, debíamos contar con los servicios de otros sistemas para asegurar que las usuarias tuvieran la atención adecuada. 


\section{Necesidades biopsicosociales de las usuarias}

Los efectos de la violencia doméstica en la salud mental en las usuarias fue un factor importante en el reporte de las consejeras. La salud mental es un estado en el que la persona utiliza sus capacidades para manejar las presiones de la vida y desenvolverse productivamente, contribuyendo de esta forma a su comunidad. Frecuentemente, de acuerdo a las participantes, las usuarias presentaban situaciones de salud mental relacionadas con una historia de trauma y violencia en su familia de origen, una experiencia reciente de abuso emocional y/o físico y múltiples problemas de relaciones familiares.

Las participantes también hablaron acerca de la falta de habilidades parentales en las usuarias, como por ejemplo una actitud abusiva o negligente hacia sus hijos. Así mismo, observaron niños que presentaron "rasgos parentales" así como niños enfrentándose con severos problemas mentales como ideaciones suicidas.

El equipo habló acerca de la falta de recursos financieros en las usuarias. La mayoría eran desempleadas y recibían asistencia pública o eran inmigrantes indocumentados que trabajaban intermitentemente con muy poca paga. Esto aunado a la falta de educación les impedía ser independientes económicamente. Las mujeres que decidían dejar a una pareja abusiva no contaban con ningún apoyo y sus opciones eran ir a un refugio o quedarse en una relación no sana.

La mayoría de las usuarias no hablaban inglés y/o se sentían más cómodas hablando en español. Las usuarias indocumentadas además de tener las necesidades biopsicosociales mencionadas anteriormente, presentaban otras dificultades como la falta de acceso a un trabajo adecuado, vivienda y atención médica. Un factor que les impedía sentirse seguras era la dificultad en obtener una Orden de Protección contra el Abuso (PFA) por falta de documentos legales que autorizaran su estancia en los Estados Unidos lo cual también las limitaba en obtener servicios de agencias federales o estatales. A pesar de que las leyes les permitían tramitar una residencia legal, 
necesitaban presentar cargos contra el victimario. La mayoría de las mujeres indocumentadas, temerosas de ser deportadas, no confiaban en los empleados gubernamentales y se quedaban en la relación abusiva.

\section{Limitaciones relacionadas con el sistema y con el programa.}

Ante la escasez de fondos, cada una de las consejeras manejaba una gran cantidad de casos. Además, muchas de ellas se sentían saturadas e incluso presentaban dramatización vicaria.

Algunos miembros del equipo reportaron dificultades en mantener límites sanos con las usuarias. Mencionaron que podría ser debido a que las consejeras querían ayudar a las usuarias y ser sus "salvadoras". A pesar de que las intenciones de las consejeras eran "estar disponibles para las usuarias todo el tiempo" esta relación podría dificultar a las usuarias ser autosuficientes y desarrollar relaciones sociales importantes.

Desafortunadamente solo existía un refugio disponible para víctimas de violencia doméstica en Filadelfia. El programa dependía de los albergues generales de la ciudad para referir a las usuarias que tenían la necesidad urgente de encontrar una vivienda alternativa. Debido a esto, no podían asegurarles una cama disponible, además de que el número de albergues en donde hablaban español era limitado. Los albergues no estaban diseñados para cubrir las necesidades de las familias que presentaban violencia doméstica por lo que las familias no tenían protección y seguían siendo vulnerables al abuso. Una dificultad adicional era que estos albergues no las proveían con una vivienda permanente al final de su estadía.

El juzgado familiar y el departamento de policía no tenían suficiente personal que hablara español y esto afectaba su capacidad de mantener a salvo a las usuarias y a sus hijos. Cuando se les pedía a los hijos que les tradujeran a las madres, los niños tenían que recrear la experiencia traumática en un contexto que no era terapéutico y a veces vivían el apoyo a su madre como una traición al padre. Esta situación ponía a los hijos en una posición 
de autoridad en donde su trabajo era asumir el rol de portavoz para obtener protección para los miembros de las familias. Otras organizaciones diseñadas para ayudar a las usuarias a interactuar con el sistema legal proveían servicios inadecuados ya que estaban sobrepasadas por la cantidad de trabajo y la falta de fondos. Las consejeras usaban gran parte de su tiempo tratando de coordinar con los sistemas externos.

\section{Descriptores de la comunidad y sus valores.}

Para poder crear un programa que capture los aspectos culturales de la comunidad, se debe explorar a través de los ojos de aquéllos que residen en ella. Afortunadamente, seis de las siete mujeres que fueron entrevistadas estaban viviendo actualmente en la comunidad o vivieron ahí en el pasado. Por lo tanto, habían experimentado los estresores que las usuarias habían enfrentado, no solo a través de los ojos de un proveedor de servicio sino a través de los ojos de un miembro de la comunidad. De acuerdo a Sánchez y Agudelo, (2008), es necesario que los trabajadores sociales reflexionen y analicen su propia realidad para lograr comprender el significado de los sistemas en los que están interviniendo.

En principio, esta comunidad (norte de Filadelfia) presentaba estresores como pobreza, violencia y falta de recursos además de que era discriminada y sufría por falta de servicios. Algunas participantes hablaron acerca de las consecuencias psicológicas relacionadas con la violencia dentro y fuera de su casa, otras hablaron de como las usuarias tienen que esperar mucho tiempo a que venga un oficial de la policía a ayudarlas cuando llaman al 911. La larga espera se atribuye a que los policías atienden otros crímenes que les parecen más serios y también a los prejuicios de lo común que les parece la violencia doméstica en el norte de Filadelfia.

Valores Culturales: Los valores culturales juegan un papel fundamental en la forma en la que los individuos experimentan su rol en el mundo y las relaciones que forman con los demás. Los valores también dictan en gran 
medida qué decisiones tomar para sí mismos y para sus familias. Guitart (2008) plantea que la participación en contextos socioculturales crea una cierta imagen de la propia identidad y los recursos necesarios para ser competentes en la sociedad. La socialización se da a través de la interiorización de pautas conductuales, normas, códigos, registros, valores y creencias.

Todas las participantes hablaron acerca de la importancia de la familia como un valor cultural en la comunidad latina. Esta concepción es un factor decisivo en su decisión de permanecer en una relación. Las mujeres sacrifican su bienestar personal para asegurarse que sus hijos tengan a sus padres presentes. La idea de proveer a los hijos con una familia intacta lleva a muchas mujeres a quedarse en una relación abusiva.

El machismo fue otro concepto cultural que mencionaron, e interesantemente, integraron en su descripción un aspecto positivo. La religión y la espiritualidad fueron otros valores relevantes en la cultura ya que muchas usuarias basaban su conducta en doctrinas dictadas por la religión o en sus creencias espirituales. Las participantes hablaron acerca de la importancia de integrar las prácticas religiosas y espirituales dentro del servicio que se les proveía a las familias para ayudarlas a desarrollar relaciones más sanas.

Características esenciales de un programa ideal: En la siguiente sección se presentan atributos particulares de un programa ideal. Las participantes hablaron acerca de la importancia de tener un programa que sea "culturalmente apropiado" y bilingüe y que sea capaz de entender las necesidades de la comunidad honrando sus valores. Una de las mayores preocupaciones fue que el programa debía de ser capaz de resolver las necesidades de las usuarias indocumentadas sin tener que depender de instancias externas.

Las participantes también hablaron acerca de tener un equipo multidisciplinario que pudiera trabajar con las familias. El equipo debe estar entrenado en violencia doméstica para entender lo que está experimentando 
una familia que acude al programa. Muchas participantes expresaron su deseo de tener un refugio en nuestra agencia que-asegurara una vivienda permanente a las usuarias. También se planteó la necesidad de alguien que representara legalmente a las usuarias y la necesidad de un consejero bilingüe que las apoye en la corte.

Debido a las dificultades financieras, un programa debe de ser capaz de proveer a las usuarias con asistencia en cuanto a sus necesidades básicas. El programa debe ofrecer cuidado de los niños y dinero para el transporte cuando las usuarias asisten a actividades programadas que no involucren a los niños. La idea de un equipo multidisciplinario fue recurrente durante las entrevistas.

Es común que surjan crisis o que las usuarias que no tienen acceso a servicios terapéuticos terminen teniendo una relación de pseudo-terapia con las consejeras. Debido a esto es importante que se encuadren las funciones de cada miembro del equipo. El equipo debe estar entrenado en trauma, intervención en crisis, empoderamiento y las etapas del ciclo de violencia.

La responsable en manejo de casos debe encargarse de: los servicios en la ciudad adonde pueda referir a las usuarias; evaluar y priorizar sus necesidades y ayudarlas a navegar los sistemas. Algunas participantes hablaron sobre la necesidad de integrar a los niños y adolescentes en el programa proveyéndolos de grupos de apoyo y alternativas para ellos. Idealmente, estas actividades deben ser provistas por la encargada de manejo de casos. Todas las actividades deben ser realizadas en un ambiente seguro y de confianza.

Las consejeras deben ser capaces de trabajar con usuarias que están en diferentes etapas de su vida (niñez, adolescencia y adultez) e integrar sus necesidades particulares a nivel individual. Deben entender cómo el proceso de migración ha afectado al bienestar de los miembros de la familia. 


\section{Tabla 2.}

Características del Programa Ideal.

\section{Programa Ideal}

\section{Caracteristicas del Programa}

Culturalmente informado

Entiende las necesidades de la

comunidad

Responde a las necesidades de la

comunidad

Provee refugios

Equipo multidisciplinario

Asistencia para necesidades básicas

Cuidado de minios

Servicios terapéuticos

Ambiente seguro y de confianza.

Entender el impacto del proceso de migración

\section{Caracteristicas de los Empleados}

Bilingüe

Entrenado en violencia domestica

Honra los valores culturales

Entiende y responde a las necesidades de las usuarias indocumentadas

Cada miembro del equipo entiende su función

Entrenamiento en trauma e intervención en crisis

Entrenamiento en las etapas de ciclo de violencia

Entrenamiento en el modelo de empoderamiento

Conocimiento de los servicios en la ciudad adonde se pueda referir a los usuarias y navegar los sistemas externos al programa.

Entender las diferentes etapas de vida de las usuarias.

\section{Discusión}

El proceso de conceptualizar y elaborar esta investigación proveyó la oportunidad de iniciar y documentar un diálogo importante entre los miembros del equipo, ya que la información obtenida podría ser relevante para desarrollar un programa culturalmente apropiado. Las respuestas de las participantes evidenciaron una serie de factores importantes en el diseño de un programa que abordara las necesidades de la comunidad. 
Una gran parte de las necesidades biopsicosociales de las usuarias se relaciona con la pobreza y la falta de seguro médico. De hecho, esto apoya la literatura actual que indica que el ingreso familiar es el mejor predictor de la violencia doméstica (Cunradi, Caetano, \& Schafer, 2000). Un aspecto resaltado por las participantes, fue la pobreza como un agravante en el mantenimiento de las relaciones abusivas. La falta de opciones se vio exacerbada en aquéllas que no hablaban inglés o que eran indocumentadas, dificultando la posibilidad de encontrar estilos de vida saludables. Gran parte de las usuarias latinas no tienen acceso a servicios de salud mental a pesar del estrés generado por la pobreza y el crimen en la comunidad. Así mismo, los trayectos migratorios traumáticos producen síntomas de estrés postraumático que no son atendidos adecuadamente. Para crear programas efectivos en la comunidad es importante tener un claro entendimiento no solo de lo que precipita el problema sino de lo que lo mantiene.

Priorizar los servicios para las familias por parte de un equipo sensibilizado e informado, debe ser uno de los pasos iniciales. A nivel comunitario, es necesario crear un conjunto de programas que provea servicios a las familias. El primer objetivo es compartir el conocimiento obtenido por esta investigación y ayudar a otros programas a incorporar un proceso similar de autorreflexión. El segundo objetivo es compartir recursos y crear una comunidad de proveedores de servicios que facilite el proceso de referir a las usuarias.

Aunque algunos de los descriptores ambientales que puntualizaron las participantes estaban asociados con la falta de recursos en la comunidad, también hablaron de la prevalencia de valores que mantuvieron a las usuarias en una relación abusiva y que juegan un papel central en la toma de decisiones.

Se destacan tres valores centrales asociados a la decisión de quedarse en la relación abusiva: familismo, marianismo y machismo. Familismo, que es el valor más arraigado por la comunidad latina, dicta la identidad de los miembros de la familia (Malley-Morrison \& Hines, 2004). Por lo tanto, la importancia de mantener a la familia unida está magnificado 
ya que sin la unidad, perderían la parte más importante de su identidad. La familia es uno de los ambientes más importantes debido a que es un sistema que transmite a sus miembros características que le dan una identidad familiar y personal, inculcando valores y patrones de comportamiento. (Castilla, Caycho, Shimabukuru \& Validivia, 2014).

Marianismo se refiere a la idea de la mujer, particularmente la madre que refleja los atributos referentes a la Virgen María. Las buenas mujeres son aquéllas que sacrifican su bienestar por el bienestar de los niños, ya que su identidad principal es ser madres (Malley-Morrison \& Hines, 2004). Las mujeres que comulgan con este valor se quedan en la relación abusiva por el bien de sus hijos. Muchas participantes mencionaron que para muchas mujeres era inconcebible terminar la relación con su pareja porque pensaban que privar a sus hijos de una figura paterna causaría un daño emocional irreparable para ellos. Consecuentemente, no apoyar este valor y abandonar a sus parejas redefine su identidad como malas mujeres, lo que pondría su bienestar personal sobre el bienestar de la familia.

El Machismo también juega un rol esencial en la decisión de las mujeres para quedarse en la relación abusiva. Machismo, como puntualizaron las participantes, tiene una connotación positiva y negativa. Por un lado demuestra que son capaces de ser proveedores financieros y dar seguridad física a los miembros de la familia. Por otro lado implica que los hombres actúan como lo que Malley-Morrison y Hines (2004) describen como hipermasculinidad que se traduce en una conducta agresiva. $Y$ es por esto que la conducta agresiva de los hombres no está mal vista por algunas mujeres e incluso es aceptada, dificultándoles dejar la relación. Es difícil separar estos tres valores y el papel que juegan en la decisión de las mujeres para quedarse ya que apoyan diferentes aspectos de cómo mantener la unión familiar.

Una de las participantes dijo que "hay que honrar" los valores que las mujeres perciben como importantes y utilizarlos para proveer servicios que sean culturalmente apropiados. Una de las mayores dificultades que enfrentó el equipo fue ser parte de un programa basado en teorías que no 
son adecuadas para la comunidad. Por ejemplo, el programa actual estaba basado en los valores estadounidenses donde el éxito se mide en cuanto a si las mujeres abandonaron la relación abusiva. Sin embargo, las necesidades y valores de las usuarias ponen un mayor énfasis en ayudar a las mujeres a mantenerse a salvo si deciden quedarse en la relación. Además, debido a que los programas siguen asumiendo que la personalidad del hombre es lo único que genera la violencia (Gelles, 1997) hay poco trabajo familiar desarrollado donde se involucre a la pareja abusiva para crear relaciones sanas.

Las participantes dijeron que un programa ideal debe proveer una amplia variedad de servicios incluyendo asistencia financiera para vivienda y acceso a servicios médicos y legales entre otras cosas. Schorr (1989) explica que atender las necesidades básicas de las usuarias es esencial para que ellas se puedan enfocar en desarrollar habilidades parentales y resolver la relación de abuso. Las participantes también dijeron que los servicios deben ser provistos en la misma agencia o en la comunidad donde la familiaridad con las usuarias facilitara el acceso. Es importante que el equipo esté entrenado en varias teorías/modelos que incluyan un entendimiento profundo de la cultura de las usuarias así como estar atentas de un posible choque entre los valores y las suposiciones de esa cultura con los de la cultura estadounidense. Esto permitirá que los servicios sean más efectivos. Las participantes subrayaron que proveer los servicios no es suficiente si éstos no son provistos en una forma que sea aceptada por la comunidad. Un programa que verdaderamente atiende las necesidades de esas usuarias provee servicios constantes sobre intervención en crisis y no solo se limita a las citas agendadas.

Finalmente las participantes subrayaron la importancia de desarrollar un programa que provea a las usuarias desde el inicio con una visión clara acerca de los servicios que provee, las funciones de los diferentes miembros del equipo y otros sistemas que pueden jugar un rol de apoyo en sus procesos. Adicionalmente, es imperativo que el equipo tenga una visión clara de las metas y prioridades de las usuarias. Esta recomendación en particular puede 
ser un desafío debido a las circunstancias por las cuales las usuarias buscaron nuestros servicios.

El reto más importante de este estudio implica ser parte de un equipo programático y mantener relaciones profesionales positivas a largo plazo con todos los miembros del equipo. A pesar de que en ciertos aspectos pareciera que esta situación facilita el desarrollo del estudio (por ejemplo, creando una mayor facilidad en el proceso de entrevista), también puede llevar a retos particulares. La familiaridad de las relaciones se extiende al proceso de entrevista y fue un desafío para la investigadora cumplir con ésta función y evitar ser compañera de trabajo o supervisora. Como compañera de trabajo y supervisora, el rol fue explorar desafíos clínicos y programáticos y ayudar al equipo a atenderlos. Fue difícil escuchar que el equipo estaba experimentando múltiples estresores y no poder atenderlos inmediatamente.

El presente estudio pretende contribuir al trabajo creciente en violencia domestica y sensibilidad cultural. Dándoles voz a aquellos que se enfrentan diariamente con las dificultades de las usuarias. Estamos parcialmente de acuerdo con San Martín y Tuya, 2014, en cuanto a la urgencia de la protección legal para las mujeres sobrevivientes de violencia domestica, así como la implementación de un nuevo enfoque en el ámbito familiar. Sin embargo, es de vital importancia considerar la devaluación de las comunidades latinas pobres tanto en Estados Unidos como en otros países de Latinoamérica. Esta devaluación e indiferencia, se ve reflejada en falta de fondos que apoyen a las necesidades de estas familias.

\section{Referencias}

Alencar-Rodrigues, R., \& Cantera, L. (2012). Violencia de género en la pareja: Una revisión teórica. Psico, 41(1), 116-126.

Bausela, E. (2003). La investigación cooperativa, una modalidad de la investigación-acción. Revista de Psicodidáctica, (15-16), 121-130. 
Bronfenbrenner, U. (1979). The ecology of human development: Experiments by nature and design. Cambridge, MA: Harvard University Press.

Castilla, H. A., Caycho, T. P., Shimabukuro, M., \& Valdivia, A. A. (2014). Percepción del funcionamiento familiar: Análisis psicométrico de la Escala APGAR-familiar en adolescentes de Lima. Propósitos y Representaciones, 2(1), 49-78. https://doi.org/10.20511/pyr2014.v2n1.53

Charmaz, K. (1995). Grounded theory. In Smith et al. (eds.), Rethinking Methods in Psychology (pp. 27-49). London: Sage Publications. https:// doi.org/10.4135/9781446221792.n3

Corsi, J. (2010). La violencia hacia las mujeres como problema social. Análisis de las consecuencias y de los factores de riesgo. Documentación de apoyo, Fundación Mujeres.

Cunradi, C.B., Caetano, R., \& Schafer, J. (2002). Socioeconomic predictors of intimate partner violence among White, Black, and Hispanic couples in the U.S. Journal of Family Violence, 17(4), 377-389. https://doi. org/10.1023/A:1020374617328

Gelles, R. (1997). Intimate violence in families ( $3^{\circ}$ ed.). Thousand Oaks, CA: Sage.

Graham-Bermann, S. A. (2001). Introduction in SA Grahamm-Bermann \& JL Edelson. Domestic violence in the lives of children: The future of research, intervention, and social policy, pp. 1-12.

Guitart, M. E. (2008). Hacia una psicología cultural. Origen, desarrollo y perspectivas. Fundamentos en Humanidades, 9(18), 7-23

Malley-Morrison, K., Hines, D. A. (2004). Family violence in a cultural perspective. Thousand Oaks, CA: Sage.

Reason, P., \& Heron, J. (1995). Co-operative inquiry. In J. A. Smith, R. Harré, \& L. V. Rethinking Methods in Psychology. https://doi.org/10.4135/9781446221792. n9

Rodríguez Franco, L., López-Cepero, J., \& Rodríguez Díaz, F. J. (2009). Violencia doméstica: una revisión bibliográfica y bibliométrica. Psicothema, 21(2), 248-254.

Sánchez, M. L. H., \& Agudelo, F. J. S. (2008). La dimensión afectiva como base del desarrollo humano una reflexión teórica para la intervención en trabajo social. Eleuthera, 2, 53-73. 
San Martín, A. U., \& Tuya, M. R. (2014). La violencia familiar un mal que se ha tornado en un problema cotidiano y que exige una solución integral. Investigaciones Sociales, 18(33), 217-226.

Schorr, L.B. (1989). Within our reach: Breaking the cycle of disadvantage. New York: Doubleday.

Torrecilla, J. M. (n.d.). La entrevista. Madrid, Espa-a: Universidad Autónoma de Madrid.

Varea, A., Manuel, J., \& Castellanos Delgado, J. L. (2006). Por un enfoque integral de la violencia familiar. Psychosocial Intervention, 15(3), 253274.

Villarejo-Aguilar, L. (1886). La teoría fundamentada en la investigación cualitativa. Revista Médica Electrónica. 


\section{Anexo A.}

\section{La Entrevista}

1. ¿Cuáles son algunas de las formas en las que has tenido dificultad en atender las necesidades de nuestras usuarias?

2. ¿Cuáles son algunas de las formas en las que el programa puede modificarse para ser más adecuado en atender mejor estas dificultades?

3. ¿Puedes hablarme de un caso que haya sido exitoso? ¿Qué es lo que te permitió ser de ayuda a la usuaria? ¿Hay algo que la agencia pudo haber hecho para ser aún más efectiva en casos como este?

4. ¿Puedes hablarme sobre un caso que haya presentado retos difíciles? ¿Que pudo haber hecho la agencia para que estuviera mejor?

5. ¿Qué aspectos de tu entrenamiento fueron particularmente de ayuda? ¿En qué crees que deberíamos proveer entrenamiento adicional?

6. ¿Basado en tu experiencia personal y en los casos en los que has trabajado, como se deben tomar en cuenta los valores de la cultura latina en casos de violencia domestica?

7. ¿Cuáles son las cosas que alguien ajeno a la cultura latina debe saber o entender? ¿Cómo el entender estos aspectos de la cultura ayuda a trabajar con las dificultades de las usuarias latinas?

8. ¿Puedes darme algunos ejemplos de casos en los que hayas trabajado en donde el hecho de que fueran de la cultura latina hizo una diferencia en lo que paso? 